Supplement of Biogeosciences, 12, 3403-3414, 2015

http://www.biogeosciences.net/12/3403/2015/

doi:10.5194/bg-12-3403-2015-supplement

(C) Author(s) 2015. CC Attribution 3.0 License.

(c) (i)

Supplement of

\title{
Differences in microbial community composition between injection and production water samples of water flooding petroleum reservoirs
}

\author{
P. K. Gao et al. \\ Correspondence to: T. Ma (tingma@nankai.edu.cn)
}

The copyright of individual parts of the supplement might differ from the CC-BY 3.0 licence. 


\section{Supplementary Text}

\section{Pyrosequencing of partial 16S rRNA genes and sequences analysis}

Broadly conserved primers 27F (5'-AGA GTT TGA TCC TGG CTC AG-3') and 533R (5'-TTA CCG CGG CTG CTG GCA C-3') containing the A and B sequencing adaptors, were used to amplify bacterial 16S rRNA gene sequences, while primers 344F (5'-ACG GGG YGC AGC AGG CGC GA-3') and 915R (5’-GTG CTC CCC CGC CAA TTC CT-3') were used to amplify archaeal 16S rRNA gene sequences. The PCRs were carried out in triplicate using $20 \mu \mathrm{L}$ reactions with $10.5 \mu \mathrm{L}$ sterile $\mathrm{ddH}_{2} \mathrm{O}, 4 \mu \mathrm{L} 5 \times \operatorname{TransStart}{ }^{\circledR F a s t P f u}$ Buffer (TransGen Biotech, China), $2 \mu \mathrm{L} 2.5 \mathrm{mM}$ dNTPs, 0.5 $\mu$ TransStart ${ }^{\circledR}$ FastPfu DNA Polymerase, $0.5 \mu \mathrm{L}$ forward primer (10 $\left.\mu \mathrm{M}\right)$, $0.5 \mu \mathrm{L}$ reverse primer $(10 \mu \mathrm{M})$, and $2 \mu \mathrm{L}$ sample DNA. For bacteria, samples were denatured for $2 \mathrm{~min}$ at $95^{\circ} \mathrm{C}$ followed by 25 cycles at $94^{\circ} \mathrm{C}$ for $30 \mathrm{~s}, 50^{\circ} \mathrm{C}$ for $30 \mathrm{~s}, 72^{\circ} \mathrm{C}$ for $30 \mathrm{~s}$ and a final elongation step at $72^{\circ} \mathrm{C}$ for $5 \mathrm{~min}$. For archaea, samples were denatured for 2 min at $95^{\circ} \mathrm{C}$ followed by 30 cycles at $94^{\circ} \mathrm{C}$ for $30 \mathrm{~s}, 50^{\circ} \mathrm{C}$ for $30 \mathrm{~s}, 72^{\circ} \mathrm{C}$ for $30 \mathrm{~s}$ and a final elongation step at $72^{\circ} \mathrm{C}$ for $5 \mathrm{~min}$.

Sequences generated from pyrosequencing were analyzed using default settings in the open source software package MOTHUR (Schloss et al., 2009). All sequences were sorted into different samples according to barcodes. Sequences were subjected to systematic checks to remove replicates, duplicates, barcodes, primer sequences and low-quality reads. Briefly, high quality sequences (> 200bp in length, quality score $>25$, exact match to barcode and primer, and containing no ambiguous characters) were remaining. These sequences were simplified using the 'unique.seqs' command to generate a unique set of sequences, and then were aligned using the 'align.seqs' command and compared with Bacterial SILVA database. The aligned sequences were clustered into OTUs with 97\% similarity.

\section{Miseq-sequencing of partial 16S rRNA genes and sequences analysis}

Pairs of reads from the original DNA fragments are merged by using FLASH (Magoc and Salzberg, 2011)-a very fast and accurate software tool which is designed to merge pairs of reads when the original DNA fragments are shorter than twice the length of reads. Sequencing reads was assigned to each sample according to the unique barcode of each sample. Raw sequences were firstly demultiplexed and then quality-filtered using the default parameters in QIIME software package (Quantitative Insights Into Microbial Ecology) and UPARSE pipeline (Caporaso et al., 2011).

First, the reads were filtered by QIIME quality filters. Default settings for Illumina processing in QIIME was used ( $r=3 p=0.75$ total read length; $\mathrm{q}=3 ; \mathrm{n}=0$ ). (p) min_per_read_length: minimum number of consecutive high-qualitybase calls to retain read(as percentage of totalread length); (r) max_bad_run_length: maximum number of consecutive low-quality base calls allowed before truncating a read; (n) sequence_max_n: maximum number of ambiguous (N) characters allowed in a sequence; (q) phred_quality_score: last quality score considered low quality

Then, we use UPARSE pipeline to picking operational taxonomic units (OTUs) through making OTU table. Sequences were assigned to OTUs at $97 \%$ similarity. The resulting representative sequence set were aligned and given a taxonomic classification using RDP (Wang et al., 2007). In order to compute Alpha Divesity, we rarify the OTU table and calculate three metrics: Chao1 metric estimates the species richness, the Observed Species metric is simply the count of unique OTUs found in the sample, and shannon index. QIIME calculates both weighted and unweighted unifrac, which were used to do Principal Coordinate Analysis (PCoA).

\section{References}

Caporaso, J. G., Lauber, C. L., Walters, W. A., Berg-Lyons, D., Lozupone, C. A., Turnbaugh, P. J., Fierer, N., and Knight, R.: Global patterns of 16S rRNA diversity at a depth of millions of sequences per sample, Proc Natl Acad Sci U S A, 108 Suppl 1, 4516-4522, 10.1073/pnas.1000080107, 2011.

Magoc, T., and Salzberg, S. L.: FLASH: fast length adjustment of short reads to improve genome assemblies, Bioinformatics, 27, 2957-2963,

10.1093/bioinformatics/btr507, 2011.

Schloss, P. D., Westcott, S. L., Ryabin, T., Hall, J. R., Hartmann, M., Hollister, E. B., Lesniewski, R. A., Oakley, B. B., Parks, D. H., Robinson, C. J., Sahl, J. W., Stres, B., Thallinger, G. G., Van Horn, D. J., and Weber, C. F.: Introducing mothur: open-source, platform-independent, community-supported software for describing and comparing microbial communities, Appl. Environ. Microbiol., 75, 7537-7541, 10.1128/AEM.01541-09, 2009.

Wang, Q., Garrity, G. M., Tiedje, J. M., and Cole, J. R.: Naïve Bayesian Classifier for Rapid Assignment of rRNA Sequences into the New Bacterial Taxonomy, Appl Environ Microb, 73, 5261-5267, 10.1128/aem.00062-07, 2007. 
Supplementary Figure: Figure S1 to Figure S3

a I
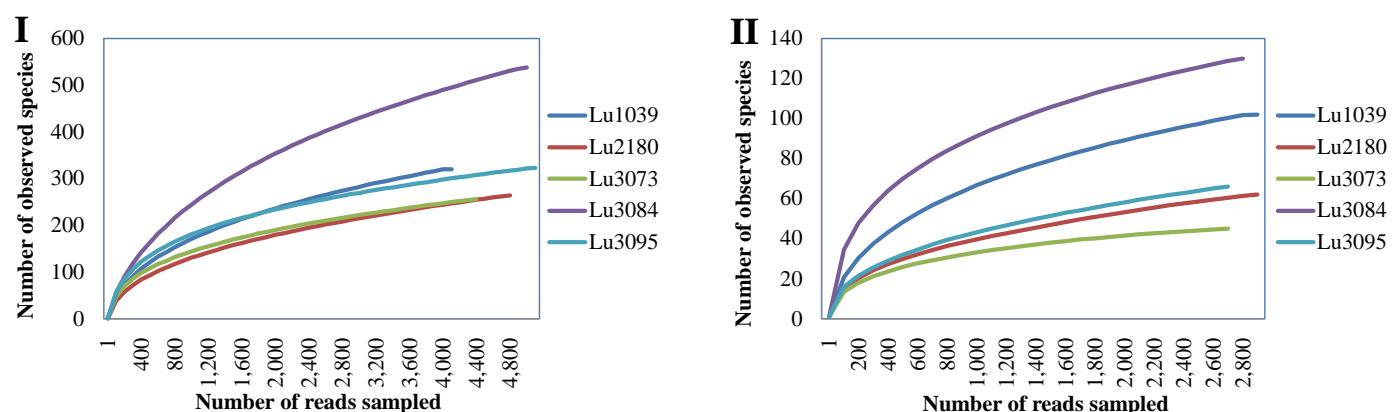

b
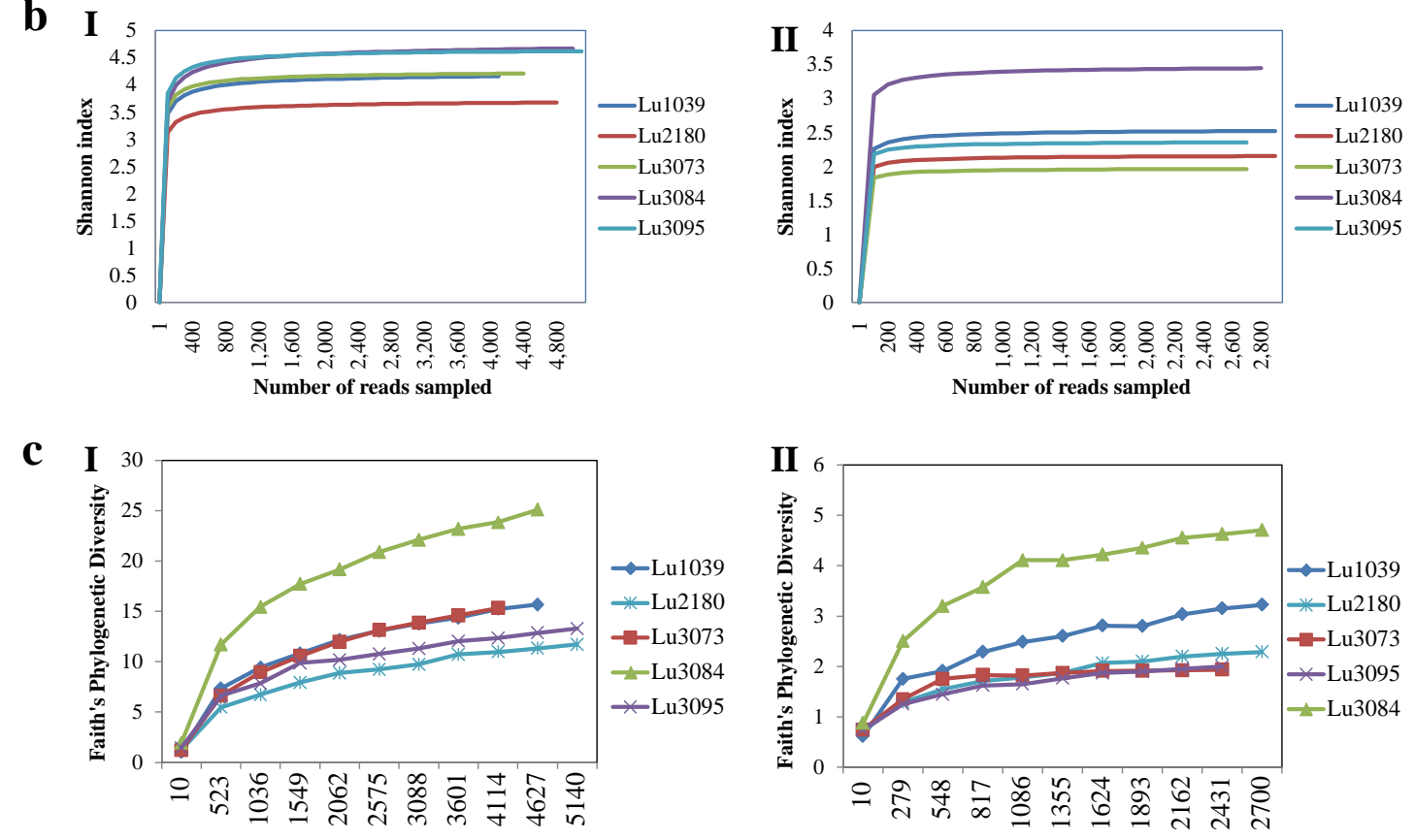

Figure S1 Rarefaction curves (a), Shannon curves (b) and phylogenetic diversity (PD) (c) of the bacterial (I) and archaeal (II) 16S rRNA gene libraries of injection and production wells in Lu-field block reservoir 


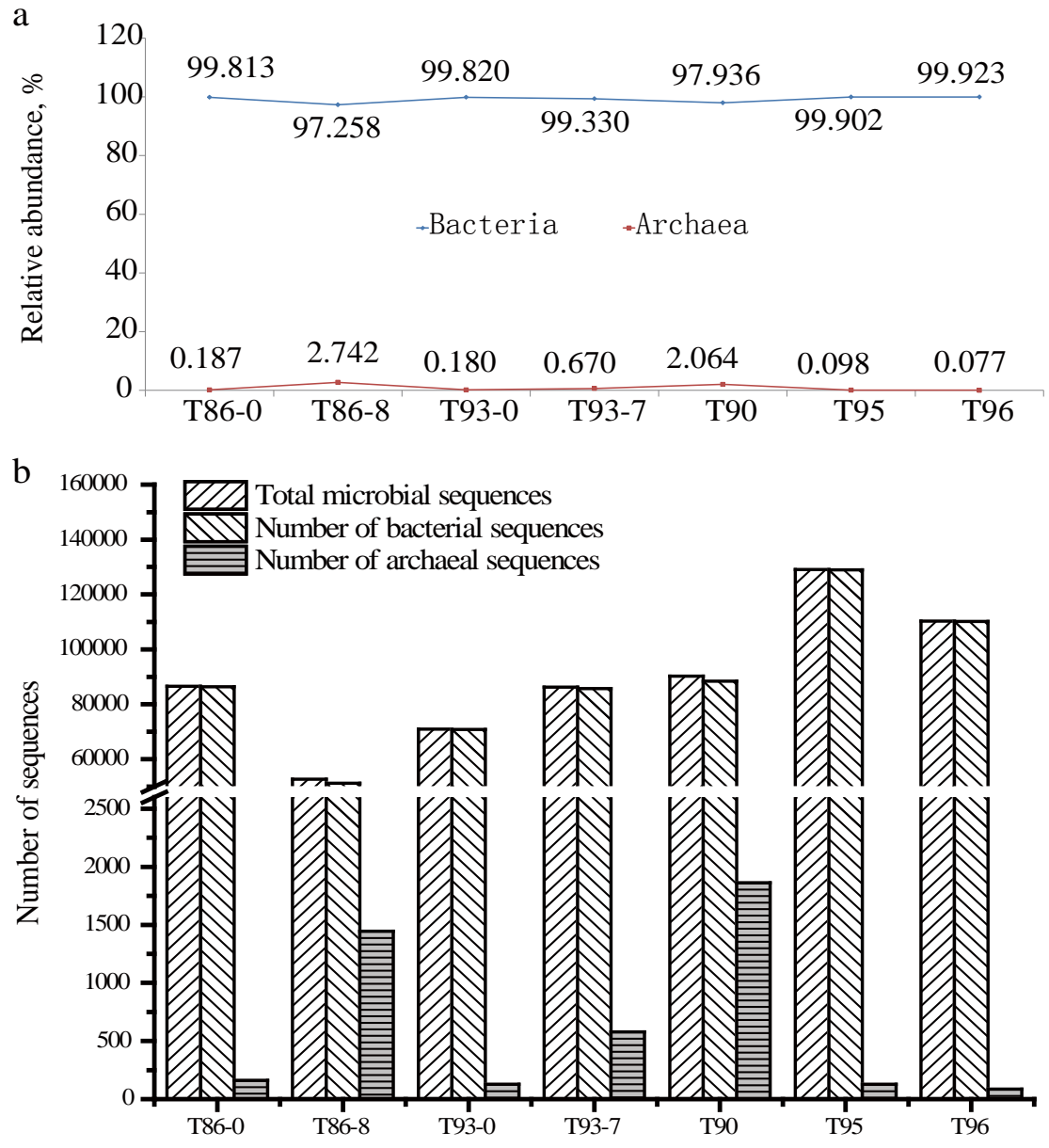

Figure S2 Statistical analyses of communities sequencing in L-field block reservoir. a, represents the relative percent of bacteria and archaea in a whole community; b, represents the number of bacterial and archaeal sequences obtained by miseq-sequencing. 

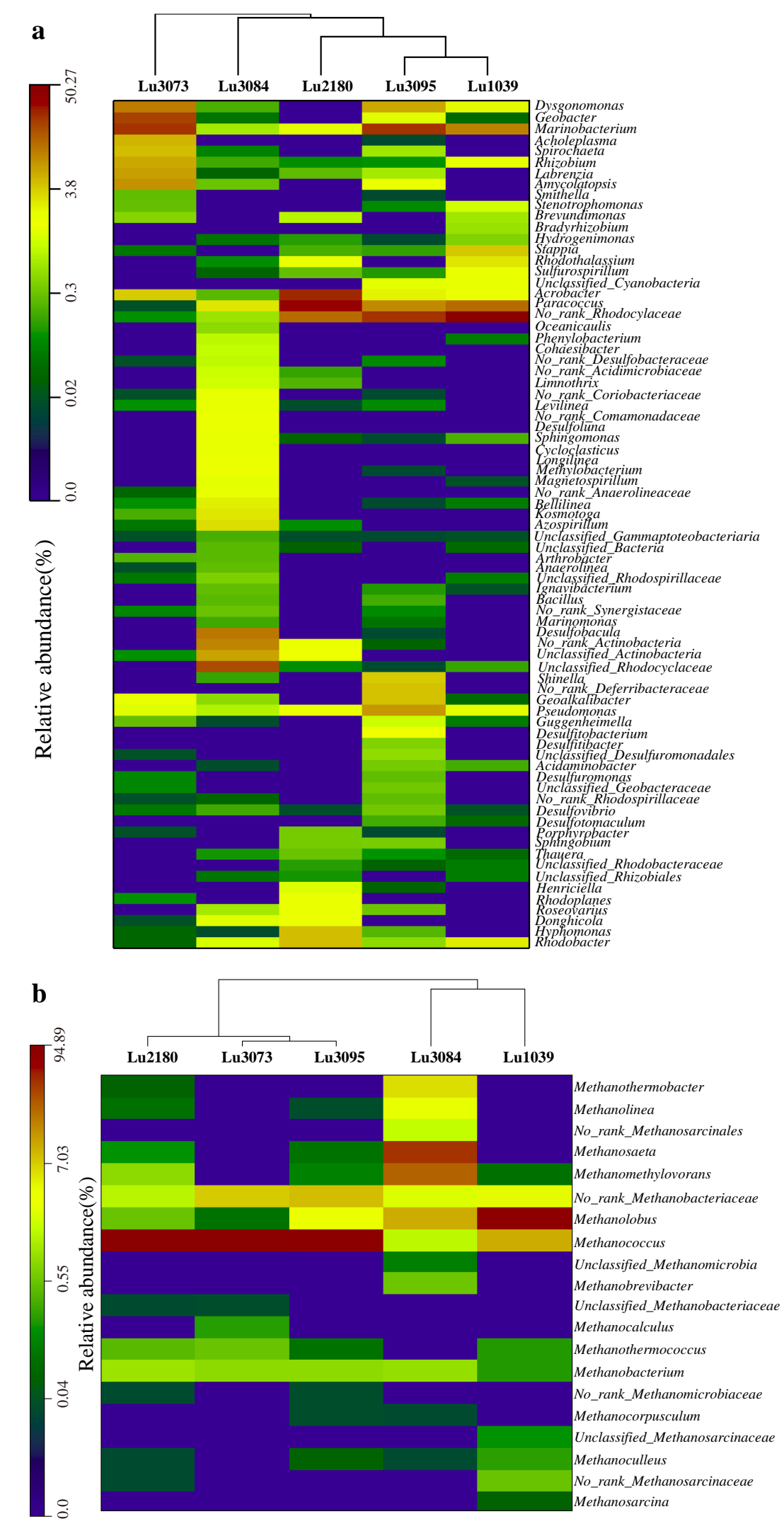

Figure S3 Heatmap showing the relative abundance and distribution of representative bacterial (a) and archaeal (b) taxa in injection and production water samples obtained from Lu-field block reservoir. 
Supplementary Table: Table S1 to S4

Table S1 Sequencing depth and alpha diversity of the microbial communities in Lu field block reservoir

\begin{tabular}{|c|c|c|c|c|c|}
\hline Library & Reads & OTUs & Chao I & Shannon & Simpson \\
\hline \multicolumn{6}{|l|}{ Bacterial } \\
\hline Lu3084 & 4957 & 538 & 892.04 & 4.67 & 0.03 \\
\hline Lu1039 & 4016 & 320 & 556.33 & 4.16 & 0.04 \\
\hline Lu2180 & 4776 & 264 & 482.90 & 3.68 & 0.07 \\
\hline Lu3073 & 4395 & 256 & 377.42 & 4.21 & 0.03 \\
\hline Lu3095 & 5060 & 323 & 486.58 & 4.62 & 0.02 \\
\hline \multicolumn{6}{|l|}{ Archaeal } \\
\hline Lu3084 & 2793 & 139 & 180.65 & 3.44 & 0.06 \\
\hline Lu1039 & 2824 & 102 & 151.4 & 2.52 & 0.15 \\
\hline Lu2180 & 2857 & 62 & 101 & 2.15 & 0.18 \\
\hline Lu3073 & 2692 & 45 & 51.11 & 1.97 & 0.23 \\
\hline Lu3095 & 2688 & 66 & 108 & 2.36 & 0.14 \\
\hline
\end{tabular}




\begin{tabular}{|c|c|c|c|c|}
\hline Library & Reads & OTUs & Chao1 & Shannon \\
\hline T86-0 & 86526 & 1555 & 4403.944 & 6.890032 \\
\hline T86-8 & 52719 & 1680 & 4439.167 & 6.896292 \\
\hline T93-0 & 70930 & 1284 & 4224.441 & 6.103269 \\
\hline T93-7 & 86256 & 1580 & 4248.355 & 6.683729 \\
\hline T90 & 90303 & 1571 & 3731.311 & 6.421689 \\
\hline T95 & 129106 & 1420 & 4256.786 & 5.956687 \\
\hline T96 & 110341 & 1284 & 4224.441 & 6.103269 \\
\hline
\end{tabular}


Table S3 The relative abundance of bacterial genera detected in Lu field block reservoir

\begin{tabular}{|c|c|c|c|c|c|}
\hline Taxa (Phylum-Class-Order-Family-Genus) & Lu3084 & Lu1039 & Lu2180 & Lu3073 & Lu3095 \\
\hline P_Actinobacteria;c_Actinobacteria;o_Actinomycetales;__Propionibacteriaceae;g_Propionibacterium & 0.04 & 0 & 0 & 0 & 0 \\
\hline p_Bacteroidetes;c_Bacteroidia;o_Bacteroidales;f_Bacteroidaceae;g_Bacteroides & 0 & 0 & 0 & 0.02 & 0.04 \\
\hline p_Bacteroidetes;c_Bacteroidia;o_Bacteroidales;f_Porphyromonadaceae;g_Paludibacter & 0 & 0 & 0 & 0.05 & 0 \\
\hline p_Bacteroidetes;c_Bacteroidia;o_Bacteroidales;f_Porphyromonadaceae;g_Proteiniphilum & 0.2 & 2.39 & 0 & 10.44 & 6.09 \\
\hline p_Bacteroidetes;c_Flavobacteriia;o_Flavobacteriales;f_Flavobacteriaceae;g_Gillisia & 0 & 0 & 0 & 0 & 0.02 \\
\hline P_Chloroflexi;c_Anaerolineae;o_Anaerolineales;f_Anaerolinaceae;g_Leptolinea & 0.28 & 0 & 0 & 0 & 0 \\
\hline p_Chloroflexi;c_Anaerolineae;o_Anaerolineales;f_Anaerolinaceae;g_Levilinea & 0.12 & 0 & 0 & 0 & 0 \\
\hline p_Chloroflexi;c_Anaerolineae;o_Anaerolineales;f_Anaerolinaceae;g_Longilinea & 0.14 & 0 & 0 & 0 & 0 \\
\hline P_Deferribacteres;c_Deferribacteres;o_Deferribacterales;f_Deferribacteraceae;g_Geovibrio & 0 & 0 & 0 & 0 & 0.06 \\
\hline P_Firmicutes;c_Clostridia;o_Clostridiales;f_Eubacteriaceae;g_Acetobacterium & 0 & 0.02 & 0 & 0 & 0.1 \\
\hline p_Firmicutes;c_Clostridia;o_Clostridiales;f_Lachnospiraceae;g_Christensenella & 0.06 & 0 & 0 & 0 & 0 \\
\hline p_Firmicutes;c_Clostridia;o_Clostridiales;_fPeptococcaceae;g_Desulfitibacter & 0 & 0 & 0 & 0 & 0.42 \\
\hline p_Firmicutes;c_Clostridia;o_Clostridiales;f_Peptococcaceae;g_Desulfitobacterium & 0 & 0 & 0 & 0 & 1.62 \\
\hline P_Firmicutes;c_Clostridia;o_Clostridiales;f_Peptococcaceae;g_Desulfosporosinus & 0 & 0 & 0 & 0 & 0.1 \\
\hline p_Firmicutes;c_Clostridia;o_Clostridiales;f_Peptococcaceae;g_Desulfurispora & 0.32 & 0 & 0 & 0 & 0 \\
\hline p_Firmicutes;c_Clostridia;o_Clostridiales;__Ruminococcaceae;g_Fastidiosipila & 0.16 & 0 & 0 & 0 & 0 \\
\hline P_Firmicutes;c_Clostridia;o_Clostridiales;g_Fusibacter & 0 & 0.07 & 0 & 0.41 & 1.09 \\
\hline p_Firmicutes;c_Erysipelotrichi;o_Erysipelotrichales;f_Erysipelotrichaceae;g_Erysipelothrix & 0 & 0 & 0 & 0 & 0.04 \\
\hline p_Proteobacteria;c_Alphaproteobacteria;o_Caulobacterales;f_Caulobacteraceae;g_Brevundimonas & 0 & 0.57 & 0.59 & 0.32 & 0 \\
\hline P_Proteobacteria;c_Alphaproteobacteria;o_Caulobacterales;f_Caulobacteraceae;g_Phenylobacterium & 0.87 & 0.02 & 0 & 0 & 0 \\
\hline P_Proteobacteria;c_Alphaproteobacteria;o_Rhizobiales;f_Bradyrhizobiaceae;g_Salinarimonas & 0 & 0 & 0 & 0 & 0.02 \\
\hline p_Proteobacteria;c_Alphaproteobacteria;o_Rhizobiales;f_Brucellaceae;g_Ochrobactrum & 0.04 & 0 & 0 & 0 & 0 \\
\hline p_Proteobacteria;c_Alphaproteobacteria;o_Rhizobiales;f_Hyphomicrobiaceae;g_Filomicrobium & 0.06 & 0 & 0 & 0 & 0 \\
\hline p_Proteobacteria;c_Alphaproteobacteria;o_Rhizobiales;f_Methylocystaceae;g_Pleomorphomonas & 0.02 & 0 & 0 & 0 & 0 \\
\hline P_Proteobacteria;c_Alphaproteobacteria;o_Rhizobiales;__Rhizobiaceae;g_Rhizobium & 0.34 & 0.07 & 0.08 & 5.94 & 4.09 \\
\hline p_Proteobacteria;c_Alphaproteobacteria;o_Rhodobacterales;f_Hyphomonadaceae;g_Hyphomonas & 0.02 & 0 & 6.16 & 0.05 & 0.28 \\
\hline p_Proteobacteria;c_Alphaproteobacteria;o_Rhodobacterales;f_Hyphomonadaceae;g_Maricaulis & 0.16 & 0 & 0 & 0 & 0 \\
\hline p_Proteobacteria;c_Alphaproteobacteria;o_Rhodobacterales;f_Hyphomonadaceae;g_Oceanicaulis & 0.46 & 0 & 0 & 0 & 0 \\
\hline P_Proteobacteria;c_Alphaproteobacteria;o_Rhodobacterales;f_Rhodobacteraceae;g_Labrenzia & 0 & 0 & 0 & 4.07 & 0 \\
\hline P_Proteobacteria;c_Alphaproteobacteria;o_Rhodobacterales;f_Rhodobacteraceae;g_Pannonibacter & 0.04 & 0 & 0.25 & 0 & 0.67 \\
\hline P_Proteobacteria;c_Alphaproteobacteria;o_Rhodobacterales;f_Rhodobacteraceae;g_Paracoccus & 0 & 0.02 & 0 & 0 & 0 \\
\hline P_Proteobacteria;c_Alphaproteobacteria;o_Rhodobacterales;f_Rhodobacteraceae;g_Rhodobacter & 0 & 0.02 & 0 & 0 & 0 \\
\hline P_Proteobacteria;c_Alphaproteobacteria;o_Rhodobacterales;f_Rhodobacteraceae;g_Rhodovulum & 0.04 & 0 & 0.02 & 0 & 0 \\
\hline p_Proteobacteria;c_Alphaproteobacteria;o_Rhodobacterales;f_Rhodobacteraceae;g_Rubellimicrobium & 0 & 0 & 0 & 0 & 0.02 \\
\hline p_Proteobacteria;c_Alphaproteobacteria;o_Rhodobacterales;f_Rhodobacteraceae;g_Stappia & 0 & 4.16 & 0.19 & 0 & 0.16 \\
\hline P_Proteobacteria;__Alphaproteobacteria;o_Rhodobacterales;__Rhodobacteraceae;g_Thioclava & 0 & 0.02 & 3.94 & 0.05 & 0 \\
\hline p_Proteobacteria;c_Alphaproteobacteria;o_Rhodospirillales;f_Rhodospirillaceae;g_Azospirillum & 2.93 & 0 & 0.08 & 0.07 & 0 \\
\hline P_Proteobacteria;c_Alphaproteobacteria;o_Rhodospirillales;f_Rhodospirillaceae;g_Caenispirillum & 0.08 & 0 & 0.06 & 0 & 0.06 \\
\hline p_Proteobacteria;c_Alphaproteobacteria;o_Rhodospirillales;f_Rhodospirillaceae;g_Magnetospirillum & 0.04 & 0 & 0 & 0 & 0 \\
\hline P_Proteobacteria;c_Alphaproteobacteria;o_Rhodospirillales;f_Rhodospirillaceae;g_Marispirillum & 0 & 0 & 0.04 & 0 & 0 \\
\hline P_Proteobacteria;c_Alphaproteobacteria;o_Rhodospirillales;f_Rhodospirillaceae;g_Novispirillum & 0.1 & 3.14 & 1.97 & 0 & 0 \\
\hline P_Proteobacteria;c_Alphaproteobacteria;o_Rhodospirillales;f_Rhodospirillaceae;g_Oceanibaculum & 0.02 & 0 & 0 & 0 & 0 \\
\hline P_Proteobacteria;c_Alphaproteobacteria;o_Rhodospirillales;_f_Rhodospirillaceae;g_Rhodospirillum & 0.28 & 0 & 0 & 0 & 0 \\
\hline P_Proteobacteria;__Alphaproteobacteria;o_Rhodospirillales;f_Rhodospirillaceae;g_Roseospirillum & 0.1 & 0 & 0 & 0.02 & 0 \\
\hline p_Proteobacteria;c_Alphaproteobacteria;o_Rhodospirillales;f_Rhodospirillaceae;g_Roseovarius & 0.59 & 0 & 1.95 & 0 & 0.32 \\
\hline P_Proteobacteria;c_Alphaproteobacteria;o_Rhodospirillales;f_Rhodospirillaceae;g_Thalassospira & 0.08 & 0 & 0 & 0 & 0 \\
\hline P_Proteobacteria;c_Alphaproteobacteria;o_Rhodospirillales;f_Rhodospirillaceae;g_Tistrella & 0.14 & 0 & 0 & 0 & 0 \\
\hline P_Proteobacteria;c_Alphaproteobacteria;o_Sphingomonadales;f_Erythrobacteraceae;g_Altererythrobacter & 0 & 0 & 0.36 & 0.02 & 0.02 \\
\hline p_Proteobacteria;c_Alphaproteobacteria;o_Sphingomonadales;f_Sphingomonadaceae;g_Novosphingobium & 0.24 & 0 & 0 & 0 & 0 \\
\hline P_Proteobacteria;c_Alphaproteobacteria;o_Sphingomonadales;f_Sphingomonadaceae;g_Sphingobium & 0 & 0 & 0.36 & 0 & 0.38 \\
\hline P_Proteobacteria;c_Alphaproteobacteria;o_Sphingomonadales;f_Sphingomonadaceae;g_Sphingomonas & 1.96 & 0 & 0 & 0 & 0 \\
\hline P_Proteobacteria;c_Betaproteobacteria;o_Burkholderiales;f_Alcaligenaceae;g_Burkholderia & 0 & 0 & 0 & 0 & 0.04 \\
\hline P_Proteobacteria;c_Betaproteobacteria;o_Burkholderiales;f_Alcaligenaceae;g_Pusillimonas & 0.1 & 0 & 0 & 0 & 0 \\
\hline P_Proteobacteria;c_Betaproteobacteria;o_Burkholderiales;f_Comamonadaceae;g_Aquabacterium & 0.06 & 0 & 0 & 0 & 0.02 \\
\hline p_Proteobacteria;c_Betaproteobacteria;o_Burkholderiales;f_Comamonadaceae;g_Brachymonas & 0.14 & 0 & 0 & 0 & 0 \\
\hline P_Proteobacteria;c_Betaproteobacteria;o_Burkholderiales;f_Comamonadaceae;g_Comamonas & 0 & 0 & 0 & 0.02 & 0.08 \\
\hline P_Proteobacteria;c_Betaproteobacteria;o_Burkholderiales;__Comamonadaceae;g_Delftia & 0 & 0 & 0 & 0 & 0.06 \\
\hline P_Proteobacteria;c_Betaproteobacteria;o_Burkholderiales;f_Comamonadaceae;g_Diaphorobacter & 0.04 & 0 & 0 & 0 & 0 \\
\hline P_Proteobacteria;c_Betaproteobacteria;o_Rhodocyclales;f_Rhodocyclaceae;g_Azoarcus & 0.02 & 0 & 0 & 0.02 & 0 \\
\hline P_Proteobacteria;c_Betaproteobacteria;o_Rhodocyclales;f_Rhodocyclaceae;g_Thauera & 0.12 & 0 & 0.06 & 0 & 0.12 \\
\hline P_Proteobacteria;c_Deltaproteobacteria;o_Desulfarculales;f_Desulfarculaceae;g_Desulfarculus & 2.2 & 0 & 0 & 0.02 & 0.1 \\
\hline P_Proteobacteria;c_Deltaproteobacteria;o_Desulfobacterales;f_Desulfobulbaceae;g_Desulfobulbus & 0.2 & 0 & 0 & 0 & 0 \\
\hline P_Proteobacteria;c_Deltaproteobacteria;o_Desulfobacterales;f_Desulfobulbaceae;g_Desulfofustis & 0.12 & 0 & 0 & 0 & 0 \\
\hline
\end{tabular}




\begin{tabular}{|c|c|c|c|c|c|}
\hline p_Proteobacteria;c_Deltaproteobacteria;o_Desulfovibrionales;f_Desulfomicrobiaceae;g_Desulfomicrobium & 0 & 0.02 & 0 & 0.05 & 0.14 \\
\hline p_Proteobacteria;c_Deltaproteobacteria;o_Desulfovibrionales;f_Desulfovibrionaceae;g_Desulfovibrio & 0.08 & 0.05 & 0.02 & 0.07 & 0.04 \\
\hline P_Proteobacteria;c_Deltaproteobacteria;o_Desulfuromonadales;f_Desulfuromonadaceae;g_Desulfuromonas & 0 & 0.02 & 0 & 11.92 & 1.48 \\
\hline p_Proteobacteria;c_Deltaproteobacteria;o_Desulfuromonadales;f_Desulfuromonadaceae;g_Donghicola & 1.09 & 0 & 1.7 & 0.02 & 0 \\
\hline P_Proteobacteria;c_Deltaproteobacteria;o_Desulfuromonadales;f_Desulfuromonadaceae;g_Pelobacter & 0.36 & 0 & 0 & 0 & 0 \\
\hline P_Proteobacteria;c_Deltaproteobacteria;o_Desulfuromonadales;f_Geobacteraceae;g_Alkalibacter & 0.04 & 0 & 0 & 0 & 0 \\
\hline P_Proteobacteria;c_Deltaproteobacteria;o_Desulfuromonadales;f_Geobacteraceae;g_Geoalkalibacter & 0 & 0.05 & 0 & 2.09 & 4.37 \\
\hline P_Proteobacteria;c_Deltaproteobacteria;o_Desulfuromonadales;f_Geobacteraceae;g_Geobacter & 0.06 & 0 & 0 & 0 & 0 \\
\hline P_Proteobacteria;c_Deltaproteobacteria;o_Syntrophobacterales;f_Desulfobacteraceae;g_Desulfococcus & 0.1 & 0 & 0 & 0 & 0 \\
\hline P_Proteobacteria;c_Deltaproteobacteria;o_Syntrophobacterales;f_Desulfobacteraceae;g_Desulfotignum & 0.28 & 0 & 0 & 0 & 0.02 \\
\hline p_Proteobacteria;c_Deltaproteobacteria;o_Syntrophobacterales;f_Syntrophaceae;g_Desulfomonile & 0.04 & 0 & 0 & 0 & 0 \\
\hline p_Proteobacteria;c_Deltaproteobacteria;o_Syntrophobacterales;__Syntrophaceae;g_Smithella & 0 & 0 & 0 & 0.27 & 0.02 \\
\hline P_Proteobacteria;c_Deltaproteobacteria;o_Syntrophobacterales;f_Syntrophobacteraceae;g_Desulfoglaeba & 0.08 & 0 & 0 & 0 & 0 \\
\hline P_Proteobacteria;c_Epsilonproteobacteria;o_Campylobacterales;__Campylobacteraceae;g_Arcobacter & 0.24 & 2.04 & 25.1 & 4.1 & 2.63 \\
\hline P_Proteobacteria;c_Epsilonproteobacteria;o_Campylobacterales;f_Campylobacteraceae;g_Sulfurospirillum & 0.04 & 2.17 & 0.4 & 0 & 0.16 \\
\hline P_Proteobacteria;c_Epsilonproteobacteria;o_Campylobacterales;f_Helicobacteraceae;g_Sulfurimonas & 0.04 & 0 & 0 & 0 & 0 \\
\hline P_Proteobacteria;c_Gammaproteobacteria;__Alteromonadales;__[Chromatiaceae];g_Alishewanella & 0.06 & 0.15 & 0 & 0 & 0 \\
\hline P_Proteobacteria;c_Gammaproteobacteria;o_Alteromonadales;f_Shewanellaceae;g_Shewanella & 0 & 0 & 0 & 0.02 & 0 \\
\hline P_Proteobacteria;c_Gammaproteobacteria;o_Oceanospirillales;f_Halomonadaceae;g_Halomonas & 0.02 & 0 & 0 & 0 & 0 \\
\hline p_Proteobacteria;c_Gammaproteobacteria;o_Oceanospirillales;f_Oceanospirillaceae;g_Marinobacterium & 0.61 & 8.42 & 1.51 & 22.3 & 22.37 \\
\hline p_Proteobacteria;c_Gammaproteobacteria;o_Oceanospirillales;f_Oceanospirillaceae;g_Marinobacterium & 0.06 & 0 & 0 & 0 & 0 \\
\hline P_Proteobacteria;c_Gammaproteobacteria;o_Oceanospirillales;f_Oceanospirillaceae;g_Nitrincola & 0 & 0 & 0.02 & 0 & 0 \\
\hline p_Proteobacteria;c_Gammaproteobacteria;o_Oceanospirillales;f_Oceanospirillaceae;g_Oceanobacter & 0.04 & 0 & 0.06 & 0.05 & 0 \\
\hline P_Proteobacteria;c_Gammaproteobacteria;o_Oceanospirillales;f_Oceanospirillaceae;g_Thalassolituus & 0.18 & 0 & 0 & 0 & 0.06 \\
\hline p_Proteobacteria;c_Gammaproteobacteria;o_Pseudomonadales;f_Pseudomonadaceae;g_Pseudomonas & 0.99 & 1.44 & 1.93 & 1.3 & 7.37 \\
\hline P_Proteobacteria;c_Gammaproteobacteria;o_Thiotrichales;f_Piscirickettsiaceae;g_Methylophaga & 0 & 0.1 & 0 & 0 & 0 \\
\hline P_Proteobacteria;c_Gammaproteobacteria;o_Xanthomonadales;__Xanthomonadaceae;g_Pseudoxanthomonas & 0 & 1.12 & 0 & 0.25 & 0.1 \\
\hline P_Spirochaetes;c_Spirochaetes;o_Spirochaetales;f_Spirochaetaceae;g_Spirochaeta & 0.08 & 0 & 0 & 4.66 & 0.61 \\
\hline P_Synergistetes;c_Synergistia;o_Synergistales;f_Aminiphilaceae;g_Aminiphilus & 0.12 & 0 & 0 & 0.05 & 0 \\
\hline P_Synergistetes;c_Synergistia;o_Synergistales;f_Thermovirgaceae;g_Thermovirga & 0.18 & 0 & 0 & 0.02 & 0.06 \\
\hline P_Tenericutes;c_Mollicutes;o_Acholeplasmatales;f_Acholeplasmataceae;g_Acholeplasma & 0 & 0 & 0 & 5.32 & 0.02 \\
\hline p_Thermi;c_Deinococci;o_Thermales;f_Thermaceae;g_Thermus & 0.02 & 0 & 0.02 & 0 & 0 \\
\hline p_Thermotogae;c_Thermotogae;o_Thermotogales;f_Thermotogaceae;g_Kosmotoga & 2.82 & 0 & 0 & 0.05 & 0 \\
\hline Total percent & 20.56 & 26.08 & 46.87 & 74.06 & 55.47 \\
\hline
\end{tabular}


Table S4 The relative abundance of bacterial genera detected in L-field block reservoir

\begin{tabular}{|c|c|c|c|c|c|c|c|}
\hline Taxa (Phylum-Class-Order-Family-Genus) & T86-0 & T86-8 & T93-0 & T93-7 & T90 & T95 & T96 \\
\hline P_Acidobacteria;c_Solibacteres;o_Solibacterales;__Solibacteraceae;g_Candidatus Solibacter & 0.0000 & 0.0000 & 0.0026 & 0.0000 & 0.0000 & 0.0180 & 0.0000 \\
\hline p_Actinobacteria;c_Actinobacteria;o_Actinomycetales;f_Cellulomonadaceae;g_Actinotalea & 0.0231 & 0.0079 & 0.0591 & 0.0258 & 0.0105 & 0.0000 & 0.0026 \\
\hline p_Actinobacteria;c_Actinobacteria;o_Actinomycetales;f_Cellulomonadaceae;g_Cellulomonas & 0.0026 & 0.0000 & 0.0051 & 0.0103 & 0.0000 & 0.0026 & 0.0000 \\
\hline p_Actinobacteria;c_Actinobacteria;o_Actinomycetales;f_Corynebacteriaceae;g_Corynebacterium & 0.0000 & 0.0079 & 0.0026 & 0.0052 & 0.0026 & 0.0103 & 0.0128 \\
\hline P_Actinobacteria;c_Actinobacteria;o_Actinomycetales;f_Dietziaceae;g_Dietzia & 0.0051 & 0.0079 & 0.0309 & 0.0103 & 0.0524 & 0.6501 & 0.8426 \\
\hline P_Actinobacteria;c_Actinobacteria;o_Actinomycetales;f_Intrasporangiaceae;g_Phycicoccus & 0.0026 & 0.0000 & 0.0000 & 0.0000 & 0.0000 & 0.0180 & 0.0000 \\
\hline p_Actinobacteria;c_Actinobacteria;o_Actinomycetales;f_Jonesiaceae;g_Jonesia & 0.0026 & 0.0000 & 0.0000 & 0.0078 & 0.0000 & 0.0000 & 0.0000 \\
\hline p_Actinobacteria;c_Actinobacteria;o_Actinomycetales;f_Microbacteriaceae;g_Leucobacter & 0.0103 & 0.0053 & 0.0900 & 0.0103 & 0.0079 & 0.0000 & 0.0000 \\
\hline p_Actinobacteria;c_Actinobacteria;o_Actinomycetales;f_Microbacteriaceae;g_Salinibacterium & 0.0051 & 0.0000 & 0.0360 & 0.0000 & 0.0000 & 0.0026 & 0.0000 \\
\hline p_Actinobacteria;c_Actinobacteria;o_Actinomycetales;f_Micrococcaceae;g_Arthrobacter & 0.0000 & 0.0053 & 0.0000 & 0.0103 & 0.0000 & 0.0000 & 0.0000 \\
\hline p_Actinobacteria;c_Actinobacteria;o_Actinomycetales;f_Mycobacteriaceae;g_Mycobacterium & 0.0000 & 0.0000 & 0.0000 & 0.0000 & 0.0026 & 0.0899 & 0.0180 \\
\hline p_Actinobacteria;c_Actinobacteria;o_Actinomycetales;f_Nocardiaceae;g_Rhodococcus & 0.0000 & 0.0000 & 0.0000 & 0.0000 & 0.0026 & 0.2518 & 3.7070 \\
\hline p_Actinobacteria;c_Actinobacteria;o_Actinomycetales;f_Nocardioidaceae;g_Aeromicrobium & 0.2237 & 0.0449 & 1.0724 & 0.0698 & 0.0760 & 0.0026 & 0.0026 \\
\hline p_Actinobacteria;c_Actinobacteria;o_Actinomycetales;f_Nocardioidaceae;g_Nocardioides & 0.0026 & 0.0026 & 0.0360 & 0.0000 & 0.0000 & 0.0000 & 0.0000 \\
\hline p_Actinobacteria;c_Actinobacteria;o_Actinomycetales;f_Propionibacteriaceae;g_Propionibacterium & 0.0000 & 0.0026 & 0.0051 & 0.0000 & 0.0105 & 0.0077 & 0.0257 \\
\hline p_Actinobacteria;c_Actinobacteria;o_Actinomycetales;f_Tsukamurellaceae;g_Tsukamurella & 0.0000 & 0.0000 & 0.0000 & 0.0000 & 0.0052 & 0.0026 & 0.0051 \\
\hline P_Actinobacteria;c_Thermoleophilia;o_Solirubrobacterales;f_Patulibacteraceae;g_Patulibacter & 0.0000 & 0.0000 & 0.0026 & 0.0000 & 0.0000 & 0.1002 & 0.0077 \\
\hline p_Bacteroidetes;c_Bacteroidia;o_Bacteroidales;f_[Odoribacteraceae];g_Butyricimonas & 0.0129 & 0.0000 & 0.0026 & 0.0000 & 0.0000 & 0.0000 & 0.0000 \\
\hline p_Bacteroidetes;c_Bacteroidia;o_Bacteroidales;f_Bacteroidaceae;g_Bacteroides & 0.9747 & 0.0897 & 2.0213 & 0.1938 & 0.0550 & 13.151 & 0.1901 \\
\hline P_Bacteroidetes;c_Bacteroidia;o_Bacteroidales;f_Porphyromonadaceae;g_Dysgonomonas & 0.0026 & 0.0079 & 0.0077 & 0.0129 & 0.0079 & 0.0180 & 0.0000 \\
\hline P_Bacteroidetes;c_Bacteroidia;o_Bacteroidales;f_Porphyromonadaceae;g_Parabacteroides & 1.9237 & 0.6150 & 1.0055 & 0.6099 & 0.1153 & 0.0360 & 0.2107 \\
\hline P_Bacteroidetes;c_Flavobacteriia;o_Flavobacteriales;f_Flavobacteriaceae;g_Aequorivita & 0.0360 & 0.0026 & 0.0257 & 0.0026 & 0.0026 & 0.0719 & 0.0077 \\
\hline P_Bacteroidetes;c_Flavobacteriia;o_Flavobacteriales;f_Flavobacteriaceae;g_Flavobacterium & 0.0154 & 0.1188 & 0.0540 & 0.0388 & 0.0052 & 0.1490 & 0.0103 \\
\hline P_Bacteroidetes;c_Flavobacteriia;o_Flavobacteriales;f_Flavobacteriaceae;g_Wautersiella & 0.0154 & 0.0000 & 0.0026 & 0.0052 & 0.0105 & 0.0000 & 0.0000 \\
\hline $\begin{array}{l}\text { p_Bacteroidetes;c_Sphingobacteriia;o_Sphingobacteriales;f_Amoebophilaceae;g_Candidatus } \\
\text { Amoebophilus }\end{array}$ & 0.0000 & 0.0000 & 0.0000 & 0.0000 & 0.0000 & 0.0000 & 0.0539 \\
\hline P_Bacteroidetes;c_Sphingobacteriia;o_Sphingobacteriales;f_Sphingobacteriaceae;g_Sphingobacterium & 0.0026 & 0.0000 & 0.0103 & 0.0000 & 0.0000 & 0.0000 & 0.0000 \\
\hline P_Chloroflexi;c_Anaerolineae;o_Anaerolineales;f_Anaerolinaceae;g_C1_B004 & 0.0077 & 0.0475 & 0.0129 & 0.0284 & 0.1048 & 0.0771 & 0.0103 \\
\hline p_Chloroflexi;c_Anaerolineae;o_Anaerolineales;f_Anaerolinaceae;g_SHD-231 & 0.0180 & 0.0977 & 0.0334 & 0.0491 & 0.1546 & 0.1824 & 0.0437 \\
\hline P_Chloroflexi;c_Anaerolineae;o_Anaerolineales;f_Anaerolinaceae;g_T78 & 0.1029 & 1.3329 & 0.0951 & 0.1706 & 2.3721 & 0.6989 & 0.2209 \\
\hline P_Chloroflexi;c_Anaerolineae;o_Anaerolineales;f_Anaerolinaceae;g_WCHB1-05 & 0.0026 & 0.0264 & 0.0000 & 0.0078 & 0.0131 & 0.0257 & 0.0000 \\
\hline P_Deferribacteres;c_Deferribacteres;o_Deferribacterales;f_Deferribacteraceae;g_Denitrovibrio & 0.0129 & 0.0106 & 0.0103 & 0.0052 & 0.0157 & 0.0000 & 0.0026 \\
\hline p_Deferribacteres;c_Deferribacteres;o_Deferribacterales;f_Deferribacteraceae;g_Flexistipes & 0.1723 & 0.1135 & 0.2083 & 0.1060 & 0.2123 & 0.0668 & 0.0719 \\
\hline p_Deferribacteres;c_Deferribacteres;o_Deferribacterales;f_Deferribacteraceae;g_Geovibrio & 0.0026 & 0.0000 & 0.0026 & 0.0052 & 0.0052 & 0.0000 & 0.0000 \\
\hline P_Fibrobacteres;c_Fibrobacteria;o_Fibrobacterales;f_Fibrobacteraceae;g_Fibrobacter & 0.0000 & 0.0000 & 0.0026 & 0.0026 & 0.0000 & 0.0231 & 0.0000 \\
\hline p_Firmicutes;c_Bacilli;o_Bacillales;f_Bacillaceae;g_Bacillus & 1.1085 & 0.7205 & 0.7509 & 0.6848 & 0.7260 & 0.1079 & 0.1284 \\
\hline p_Firmicutes;c_Bacilli;o_Bacillales;f_Bacillaceae;g_Virgibacillus & 0.6841 & 0.3721 & 0.3317 & 0.4264 & 0.4063 & 0.0000 & 0.0000 \\
\hline p_Firmicutes;c_Bacilli;o_Bacillales;f_Paenibacillaceae;g_Brevibacillus & 0.0000 & 0.0000 & 0.0000 & 0.0000 & 0.0000 & 0.0206 & 0.0051 \\
\hline P_Firmicutes;c_Bacilli;o_Bacillales;f_Paenibacillaceae;g_Cohnella & 0.0051 & 0.0000 & 0.0051 & 0.0026 & 0.0000 & 0.0000 & 0.0000 \\
\hline p_Firmicutes;c_Bacilli;o_Bacillales;f_Paenibacillaceae;g_Paenibacillus & 0.0000 & 0.0000 & 0.0103 & 0.0026 & 0.0026 & 0.1002 & 0.0719 \\
\hline P_Firmicutes;c_Bacilli;o_Lactobacillales;f_Aerococcaceae;g_Facklamia & 0.0926 & 0.0370 & 0.1440 & 0.2223 & 0.0315 & 0.0000 & 0.0000 \\
\hline p_Firmicutes;c_Bacilli;o_Lactobacillales;f_Carnobacteriaceae;g_Trichococcus & 0.0077 & 0.0264 & 0.0180 & 0.0594 & 0.0026 & 1.4903 & 0.1696 \\
\hline p_Firmicutes;c_Clostridia;o_Clostridiales;f_Eubacteriaceae;g_Acetobacterium & 0.0180 & 0.0185 & 0.0180 & 0.0078 & 0.0105 & 0.0822 & 0.0514 \\
\hline p_Firmicutes;c_Clostridia;o_Clostridiales;f_Lachnospiraceae;g_Anaerostipes & 0.0051 & 0.0026 & 0.0000 & 0.0310 & 0.0026 & 0.0308 & 0.0000 \\
\hline p_Firmicutes;c_Clostridia;o_Clostridiales;f_Peptococcaceae;g_Dehalobacter & 0.0103 & 0.0633 & 0.0026 & 0.0181 & 0.0157 & 0.0000 & 0.0000 \\
\hline p_Firmicutes;c_Clostridia;o_Clostridiales;f_Peptococcaceae;g_Desulfitobacterium & 0.0026 & 0.0290 & 0.0051 & 0.0026 & 0.0026 & 0.0000 & 0.0000 \\
\hline p_Firmicutes;c_Clostridia;o_Clostridiales;f_Peptococcaceae;g_Desulfosporosinus & 0.0051 & 0.0000 & 0.0026 & 0.0000 & 0.0000 & 0.0103 & 0.0000 \\
\hline p_Firmicutes;c_Clostridia;o_Clostridiales;f_Peptococcaceae;g_Desulfotomaculum & 0.0000 & 0.0000 & 0.0000 & 0.0000 & 0.0000 & 0.0231 & 0.0026 \\
\hline P_Firmicutes;c_Clostridia;o_Clostridiales;f_Peptococcaceae;g_Desulfurispora & 0.0026 & 0.0026 & 0.0000 & 0.0000 & 0.0000 & 0.0051 & 0.0026 \\
\hline P_Firmicutes;c_Clostridia;o_Clostridiales;f_Peptococcaceae;g_Niigata-25 & 0.0231 & 0.0317 & 0.0283 & 0.0594 & 0.0131 & 0.0257 & 0.0026 \\
\hline P_Firmicutes;c_Clostridia;o_Clostridiales;__Peptococcaceae;g_Pelotomaculum & 0.0000 & 0.0000 & 0.0000 & 0.0000 & 0.0000 & 0.0077 & 0.0051 \\
\hline P_Firmicutes;c_Clostridia;o_Clostridiales;f_Peptococcaceae;g_SJA-118 & 0.0000 & 0.0000 & 0.0000 & 0.0000 & 0.0000 & 0.0103 & 0.0000 \\
\hline P_Firmicutes;c_Clostridia;o_Clostridiales;f_Peptococcaceae;g_Sporotomaculum & 0.0000 & 0.0000 & 0.0000 & 0.0000 & 0.0000 & 0.0154 & 0.0026 \\
\hline P_Firmicutes;c_Clostridia;o_Clostridiales;f_Peptostreptococcaceae;g_Peptostreptococcus & 0.0026 & 0.0000 & 0.0026 & 0.0078 & 0.0000 & 0.0000 & 0.0000 \\
\hline P_Firmicutes;c_Clostridia;o_Clostridiales;f_Ruminococcaceae;g_Anaerofilum & 0.0051 & 0.0026 & 0.0051 & 0.0052 & 0.0026 & 0.3186 & 0.0334 \\
\hline P_Firmicutes;c_Clostridia;o_Clostridiales;f_Ruminococcaceae;g_Anaerotruncus & 0.0000 & 0.0000 & 0.0026 & 0.0000 & 0.0079 & 0.0000 & 0.0000 \\
\hline P_Firmicutes;c_Clostridia;o_Clostridiales;f_Ruminococcaceae;g_Oscillospira & 0.0463 & 0.0581 & 0.0283 & 0.0413 & 0.0262 & 0.0000 & 0.0000 \\
\hline P_Firmicutes;c_Clostridia;o_Clostridiales;f_Ruminococcaceae;g_Ruminococcus & 0.0746 & 0.3167 & 0.0900 & 0.3360 & 0.0813 & 0.0360 & 0.0128 \\
\hline P_Firmicutes;c_Clostridia;o_Clostridiales;f_Syntrophomonadaceae;g_Syntrophomonas & 0.0129 & 0.1980 & 0.0180 & 0.0207 & 0.0603 & 0.0026 & 0.0000 \\
\hline p_Firmicutes;c_Clostridia;o_Clostridiales;f_Veillonellaceae;g_Acidaminococcus & 0.0000 & 0.0053 & 0.0026 & 0.0284 & 0.0026 & 0.0077 & 0.0000 \\
\hline p_Firmicutes;c_Clostridia;o_Clostridiales;f_Veillonellaceae;g_vadinHB04 & 0.0257 & 0.1795 & 0.0334 & 0.5582 & 0.0367 & 0.1773 & 0.0334 \\
\hline P_Firmicutes;c_Clostridia;o_Desulfitobacterales;f_Desulfitobacteraceae;g_Desulfitobacter & 0.0077 & 0.0713 & 0.0180 & 0.0078 & 0.0131 & 0.0000 & 0.0000 \\
\hline P_Firmicutes;c_Erysipelotrichi;o_Erysipelotrichales;f_Erysipelotrichaceae;g_Erysipelothrix & 0.0026 & 0.0053 & 0.0000 & 0.0052 & 0.0000 & 0.0000 & 0.0000 \\
\hline
\end{tabular}




\begin{tabular}{|c|c|c|c|c|c|c|c|}
\hline P_Firmicutes;c_Erysipelotrichi;o_Erysipelotrichales;f_Erysipelotrichaceae;g_PSB-M-3 & 0.0051 & 0.0211 & 0.0026 & 0.0078 & 0.0105 & 0.0000 & 0.0000 \\
\hline P_Nitrospirae;c_Nitrospira;o_Nitrospirales;f_Thermodesulfovibrionaceae;g_Thermodesulfovibrio & 0.0386 & 0.0026 & 0.0411 & 0.0078 & 0.0026 & 0.0026 & 0.0000 \\
\hline p_Planctomycetes;c_Planctomycetia;o_Gemmatales;f_Gemmataceae;g_Gemmata & 0.0000 & 0.0000 & 0.0000 & 0.0000 & 0.0000 & 0.0206 & 0.0026 \\
\hline P_Planctomycetes;c_Planctomycetia;o_Planctomycetales;f_Planctomycetaceae;g_Planctomyces & 0.0000 & 0.0000 & 0.0051 & 0.0078 & 0.0026 & 0.0077 & 0.0000 \\
\hline P_Proteobacteria;c_Alphaproteobacteria;o_Caulobacterales;f_Caulobacteraceae;g_Brevundimonas & 0.0051 & 0.0000 & 0.0000 & 0.0052 & 0.0000 & 0.2004 & 0.2954 \\
\hline p_Proteobacteria;c_Alphaproteobacteria;o_Caulobacterales;f_Caulobacteraceae;g_Mycoplana & 0.0000 & 0.0000 & 0.0000 & 0.0000 & 0.0000 & 0.4625 & 0.0411 \\
\hline P_Proteobacteria;c_Alphaproteobacteria;o_Caulobacterales;__Caulobacteraceae;g_Phenylobacterium & 0.0026 & 0.0000 & 0.0051 & 0.0000 & 0.0052 & 0.0308 & 0.0000 \\
\hline P_Proteobacteria;c_Alphaproteobacteria;o_Kiloniellales;f_Kiloniellaceae;g_Thalassospira & 0.1312 & 0.0158 & 0.0051 & 0.0078 & 0.0105 & 0.0180 & 0.0334 \\
\hline P_Proteobacteria;c_Alphaproteobacteria;o_Rhizobiales;f_Brucellaceae;g_Ochrobactrum & 0.8281 & 0.4302 & 0.8769 & 0.6590 & 0.6055 & 0.2878 & 0.2261 \\
\hline P_Proteobacteria;c_Alphaproteobacteria;o_Rhizobiales;f_Hyphomicrobiaceae;g_Devosia & 0.0180 & 0.0053 & 0.0951 & 0.0181 & 0.0157 & 0.1053 & 0.0411 \\
\hline P_Proteobacteria;c_Alphaproteobacteria;o_Rhizobiales;f_Hyphomicrobiaceae;g_Hyphomicrobium & 0.0000 & 0.0026 & 0.0077 & 0.0000 & 0.0000 & 0.0051 & 0.0000 \\
\hline P_Proteobacteria;c_Alphaproteobacteria;o_Rhizobiales;f_Hyphomicrobiaceae;g_Parvibaculum & 0.1003 & 0.0079 & 0.0823 & 0.0078 & 0.0472 & 1.3104 & 0.4650 \\
\hline P_Proteobacteria;c_Alphaproteobacteria;o_Rhizobiales;f_Hyphomicrobiaceae;g_Rhodoplanes & 0.0000 & 0.0000 & 0.0077 & 0.0000 & 0.0000 & 0.0026 & 0.0000 \\
\hline P_Proteobacteria;c_Alphaproteobacteria;o_Rhizobiales;f_Methylobacteriaceae;g_Methylobacterium & 0.0000 & 0.0000 & 0.0000 & 0.0000 & 0.0000 & 0.0103 & 0.0206 \\
\hline P_Proteobacteria;c_Alphaproteobacteria;o_Rhizobiales;f_Phyllobacteriaceae;g_Aminobacter & 0.0026 & 0.0026 & 0.0077 & 0.0026 & 0.0000 & 0.0051 & 0.0000 \\
\hline p_Proteobacteria;c_Alphaproteobacteria;o_Rhizobiales;f_Rhizobiaceae;g_Agrobacterium & 0.0103 & 0.0079 & 0.0977 & 0.0207 & 0.0052 & 0.1182 & 0.1002 \\
\hline p_Proteobacteria;c_Alphaproteobacteria;o_Rhodobacterales;f_Hyphomonadaceae;g_Hyphomonas & 0.0051 & 0.0053 & 0.0103 & 0.0155 & 0.0708 & 0.5267 & 0.3699 \\
\hline P_Proteobacteria;c_Alphaproteobacteria;o_Rhodobacterales;f_Hyphomonadaceae;g_Maricaulis & 0.0000 & 0.0000 & 0.0000 & 0.0000 & 0.0000 & 0.0874 & 0.0437 \\
\hline P_Proteobacteria;c_Alphaproteobacteria;o_Rhodobacterales;f_Hyphomonadaceae;g_Oceanicaulis & 0.0000 & 0.0000 & 0.0000 & 0.0000 & 0.0000 & 0.1182 & 0.0591 \\
\hline P_Proteobacteria;c_Alphaproteobacteria;o_Rhodobacterales;f_Rhodobacteraceae;g_Antarctobacter & 0.0540 & 0.1056 & 0.0797 & 0.0388 & 0.1992 & 0.0976 & 0.0796 \\
\hline P_Proteobacteria;c_Alphaproteobacteria;o_Rhodobacterales;f_Rhodobacteraceae;g_Paracoccus & 1.4711 & 0.4276 & 0.9772 & 1.7651 & 0.4141 & 0.0077 & 0.0308 \\
\hline p_Proteobacteria;c_Alphaproteobacteria;o_Rhodobacterales;f_Rhodobacteraceae;g_Rhodobacter & 0.0000 & 0.0026 & 0.0000 & 0.0000 & 0.0000 & 0.0283 & 0.0231 \\
\hline P_Proteobacteria;c_Alphaproteobacteria;o_Rhodobacterales;f_Rhodobacteraceae;g_Rhodovulum & 0.0000 & 0.0026 & 0.0129 & 0.0000 & 0.0000 & 0.1259 & 0.0360 \\
\hline p_Proteobacteria;c_Alphaproteobacteria;o_Rhodospirillales;f_Acetobacteraceae;g_Roseomonas & 0.0026 & 0.0000 & 0.0000 & 0.0000 & 0.0000 & 0.0385 & 0.0154 \\
\hline P_Proteobacteria;c_Alphaproteobacteria;o_Rhodospirillales;f_Rhodospirillaceae;g_Azospirillum & 0.0000 & 0.0026 & 0.0026 & 0.0000 & 0.0000 & 0.0591 & 0.0822 \\
\hline p_Proteobacteria;c_Alphaproteobacteria;o_Rhodospirillales;f_Rhodospirillaceae;g_Magnetospirillum & 0.0000 & 0.0000 & 0.0000 & 0.0000 & 0.0000 & 0.0437 & 0.0308 \\
\hline p_Proteobacteria;c_Alphaproteobacteria;o_Rhodospirillales;f_Rhodospirillaceae;g_Novispirillum & 0.1157 & 0.0845 & 0.1414 & 0.1344 & 0.1756 & 0.1747 & 0.1259 \\
\hline P_Proteobacteria;c_Alphaproteobacteria;o_Rhodospirillales;f_Rhodospirillaceae;g_Phaeospirillum & 0.0000 & 0.0000 & 0.0000 & 0.0000 & 0.0000 & 0.0719 & 0.0103 \\
\hline p_Proteobacteria;c_Alphaproteobacteria;o_Sphingomonadales;f_Erythrobacteraceae;g_Erythrobacter & 0.0000 & 0.0000 & 0.0000 & 0.0000 & 0.0000 & 0.0206 & 0.0026 \\
\hline p_Proteobacteria;c_Alphaproteobacteria;o_Sphingomonadales;f_Erythrobacteraceae;g_Lutibacterium & 0.0000 & 0.0000 & 0.0077 & 0.0026 & 0.0000 & 0.0000 & 0.0000 \\
\hline $\begin{array}{l}\text { p_Proteobacteria;c_Alphaproteobacteria;o_Sphingomonadales;f_Sphingomonadaceae;g_Novosphingobiu } \\
\text { m }\end{array}$ & 0.0000 & 0.0053 & 0.0154 & 0.0362 & 0.0079 & 0.0668 & 0.0257 \\
\hline p_Proteobacteria;c_Alphaproteobacteria;o_Sphingomonadales;f_Sphingomonadaceae;g_Sphingobium & 0.0026 & 0.0026 & 0.0951 & 0.0026 & 0.0052 & 0.0000 & 0.0026 \\
\hline p_Proteobacteria;c_Alphaproteobacteria;o_Sphingomonadales;f_Sphingomonadaceae;g_Sphingomonas & 0.0000 & 0.0026 & 0.0154 & 0.0026 & 0.0052 & 0.1542 & 0.0899 \\
\hline p_Proteobacteria;c_Alphaproteobacteria;o_Sphingomonadales;f_Sphingomonadaceae;g_Sphingopyxis & 0.0103 & 0.0000 & 0.0051 & 0.0026 & 0.0000 & 0.1722 & 0.0103 \\
\hline p_Proteobacteria;c_Betaproteobacteria;o_Burkholderiales;f_Alcaligenaceae;g_Achromobacter & 0.0823 & 0.0343 & 0.0643 & 0.0569 & 0.0524 & 0.0154 & 0.0257 \\
\hline P_Proteobacteria;c_Betaproteobacteria;o_Burkholderiales;f_Alcaligenaceae;g_Denitrobacter & 0.0026 & 0.0000 & 0.0000 & 0.0078 & 0.0000 & 0.0000 & 0.0000 \\
\hline p_Proteobacteria;c_Betaproteobacteria;o_Burkholderiales;f_Alcaligenaceae;g_Pigmentiphaga & 0.0000 & 0.0000 & 0.0051 & 0.0000 & 0.0000 & 0.0103 & 0.0180 \\
\hline P_Proteobacteria;c_Betaproteobacteria;o_Burkholderiales;f_Comamonadaceae;g_Aquabacterium & 0.0231 & 0.1029 & 0.0154 & 0.0129 & 0.0026 & 0.0103 & 0.1310 \\
\hline P_Proteobacteria;c_Betaproteobacteria;o_Burkholderiales;f_Comamonadaceae;g_Hydrogenophaga & 0.0000 & 0.0000 & 0.0000 & 0.0000 & 0.0000 & 0.0797 & 0.0206 \\
\hline p_Proteobacteria;c_Betaproteobacteria;o_Hydrogenophilales;f_Hydrogenophilaceae;g_Thiobacillus & 0.0386 & 0.0554 & 0.0283 & 0.0439 & 0.0603 & 0.1644 & 0.0154 \\
\hline P_Proteobacteria;c_Betaproteobacteria;o_Procabacteriales;f_Procabacteriaceae;g_Procabacter & 0.0000 & 0.0000 & 0.0000 & 0.0000 & 0.0000 & 0.0000 & 0.0411 \\
\hline P_Proteobacteria;c_Betaproteobacteria;o_Rhodocyclales;__Rhodocyclaceae;g_Azovibrio & 0.0103 & 0.0132 & 0.0103 & 0.0078 & 0.0288 & 0.0334 & 0.0565 \\
\hline P_Proteobacteria;c_Betaproteobacteria;o_Rhodocyclales;f_Rhodocyclaceae;g_Dechloromonas & 0.0463 & 0.0633 & 0.0309 & 0.1318 & 0.0157 & 0.0051 & 0.0000 \\
\hline p_Proteobacteria;c_Betaproteobacteria;o_Rhodocyclales;f_Rhodocyclaceae;g_Hydrogenophilus & 0.0026 & 0.0000 & 0.0000 & 0.0103 & 0.0000 & 0.0026 & 0.0026 \\
\hline P_Proteobacteria;c_Betaproteobacteria;o_Rhodocyclales;f_Rhodocyclaceae;g_Thauera & 1.9392 & 1.4780 & 0.9155 & 1.2715 & 0.5006 & 0.0360 & 0.0617 \\
\hline P_Proteobacteria;c_Betaproteobacteria;o_Rhodocyclales;f_Rhodocyclaceae;g_Z-35 & 0.0026 & 0.0000 & 0.0026 & 0.0000 & 0.0000 & 0.0026 & 0.0026 \\
\hline P_Proteobacteria;c_Deltaproteobacteria;o_Bdellovibrionales;f_Bacteriovoracaceae;g_Bacteriovorax & 0.0051 & 0.0053 & 0.0283 & 0.0052 & 0.0079 & 0.0745 & 0.1978 \\
\hline P_Proteobacteria;c_Deltaproteobacteria;o_Desulfobacterales;f_Desulfobulbaceae;g_Desulfobulbus & 0.0283 & 0.1557 & 0.0463 & 0.1783 & 0.0524 & 0.0103 & 0.0077 \\
\hline $\begin{array}{l}\text { P_Proteobacteria;c_Deltaproteobacteria;o_Desulfovibrionales;f_Desulfomicrobiaceae;g_Desulfomicrobiu } \\
\mathrm{m}\end{array}$ & 0.0643 & 0.1293 & 0.0694 & 0.2300 & 0.0681 & 0.0283 & 0.0257 \\
\hline p_Proteobacteria;c_Deltaproteobacteria;o_Desulfovibrionales;f_Desulfovibrionaceae;g_Desulfovibrio & 0.3883 & 0.1029 & 0.1697 & 0.1240 & 0.1651 & 0.3340 & 0.2338 \\
\hline $\begin{array}{l}\text { P_Proteobacteria;c_Deltaproteobacteria;o_Desulfuromonadales;f_Desulfuromonadaceae;g_Desulfuromon } \\
\text { as }\end{array}$ & 0.0000 & 0.0000 & 0.0000 & 0.0026 & 0.0000 & 0.0077 & 0.0360 \\
\hline p_Proteobacteria;c_Deltaproteobacteria;o_Syntrophobacterales;f_Desulfobacteraceae;g_Desulfobacter & 0.0411 & 0.0475 & 0.0591 & 0.7262 & 0.0393 & 0.0026 & 0.0026 \\
\hline P_Proteobacteria;c_Deltaproteobacteria;o_Syntrophobacterales;f_Desulfobacteraceae;g_Desulfobacterium & 0.0000 & 0.0053 & 0.0000 & 0.0000 & 0.0210 & 0.0077 & 0.0000 \\
\hline p_Proteobacteria;c_Deltaproteobacteria;o_Syntrophobacterales;f_Desulfobacteraceae;g_Desulfococcus & 0.0000 & 0.0106 & 0.0000 & 0.0026 & 0.0131 & 0.0026 & 0.0000 \\
\hline p_Proteobacteria;c_Deltaproteobacteria;o_Syntrophobacterales;f_Desulfobacteraceae;g_Desulfotignum & 0.1415 & 0.1927 & 0.1517 & 0.1835 & 0.4508 & 0.0463 & 0.0617 \\
\hline P_Proteobacteria;c_Deltaproteobacteria;o_Syntrophobacterales;f_Syntrophaceae;g_Syntrophus & 0.0180 & 0.0185 & 0.0077 & 0.0155 & 0.0236 & 0.0051 & 0.0000 \\
\hline $\begin{array}{l}\text { p_Proteobacteria;c_Deltaproteobacteria;o_Syntrophobacterales;f_Syntrophobacteraceae;g_Syntrophobacte } \\
\text { r }\end{array}$ & 0.0000 & 0.0000 & 0.0000 & 0.0000 & 0.0000 & 0.0103 & 0.0051 \\
\hline p_Proteobacteria;c_Epsilonproteobacteria;o_Campylobacterales;f_Campylobacteraceae;g_Arcobacter & 0.6352 & 1.7974 & 0.7201 & 0.7288 & $\begin{array}{r}10.539 \\
4\end{array}$ & $\begin{array}{r}22.159 \\
4\end{array}$ & $\begin{array}{r}14.263 \\
0\end{array}$ \\
\hline $\begin{array}{l}\text { P_Proteobacteria;c_Epsilonproteobacteria;o_Campylobacterales;f_Campylobacteraceae;g_Sulfurospirillu } \\
\mathrm{m}\end{array}$ & 0.3266 & 0.3510 & 0.4063 & 0.3334 & 1.2136 & 0.0437 & 0.1387 \\
\hline
\end{tabular}




\begin{tabular}{|c|c|c|c|c|c|c|c|}
\hline p_Proteobacteria;c_Epsilonproteobacteria;o_Campylobacterales;f_Helicobacteraceae;g_Sulfurimonas & 0.1363 & 0.4988 & 0.1440 & 0.6332 & 0.1179 & 0.2518 & 3.0108 \\
\hline P_Proteobacteria;c_Gammaproteobacteria;o_Alteromonadales;__[Chromatiaceae];g_Alishewanella & 0.0489 & 0.2798 & 0.0514 & 0.1111 & 0.0472 & 0.0642 & 0.3622 \\
\hline P_Proteobacteria;c_Gammaproteobacteria;o_Alteromonadales;f_Alteromonadaceae;g_HB2-32-21 & 0.0411 & 0.0264 & 0.3240 & 0.0413 & 0.0734 & 0.0000 & 0.0000 \\
\hline P_Proteobacteria;c_Gammaproteobacteria;o_Alteromonadales;f_Alteromonadaceae;g_Marinobacter & 0.7535 & 0.3484 & 0.4320 & 0.4574 & 2.9094 & 1.8706 & 4.9016 \\
\hline p_Proteobacteria;c_Gammaproteobacteria;o_Alteromonadales;f_Alteromonadaceae;g_ZD0117 & 0.0231 & 0.0106 & 0.1569 & 0.0181 & 0.0157 & 0.0000 & 0.0000 \\
\hline P_Proteobacteria;c_Gammaproteobacteria;o_Alteromonadales;f_Idiomarinaceae;g_Pseudidiomarina & 0.3240 & 0.5912 & 0.5658 & 0.2998 & 0.0996 & 0.3674 & 0.7245 \\
\hline P_Proteobacteria;c_Gammaproteobacteria;o_Alteromonadales;f_Shewanellaceae;g_Shewanella & 0.0386 & 0.0818 & 0.0360 & 0.0388 & 0.0262 & 0.8608 & 10.859 \\
\hline P_Proteobacteria;c_Gammaproteobacteria;o_Chromatiales;__Halothiobacillaceae;g_Halothiobacillus & 0.0000 & 0.0000 & 0.0000 & 0.0000 & 0.0000 & 0.0000 & 0.0617 \\
\hline P_Proteobacteria;c_Gammaproteobacteria;o_Chromatiales;f_Halothiobacillaceae;g_Thiofaba & 0.0103 & 0.0000 & 0.0103 & 0.0000 & 0.0026 & 0.0026 & 0.0051 \\
\hline P_Proteobacteria;c_Gammaproteobacteria;o_Enterobacteriales;f_Enterobacteriaceae;g_Escherichia & 0.0129 & 0.0053 & 0.0411 & 0.0646 & 0.0341 & 0.0051 & 0.0026 \\
\hline P_Proteobacteria;c_Gammaproteobacteria;__Enterobacteriales;f_Enterobacteriaceae;g_Morganella & 0.0129 & 0.0000 & 0.0077 & 0.0026 & 0.0026 & 0.0000 & 0.0000 \\
\hline P_Proteobacteria;c_Gammaproteobacteria;o_Enterobacteriales;f_Enterobacteriaceae;g_Trabulsiella & 0.0077 & 0.0106 & 0.0103 & 0.0388 & 0.0026 & 0.3829 & 1.1201 \\
\hline p_Proteobacteria;c_Gammaproteobacteria;o_Oceanospirillales;f_Alcanivoracaceae;g_Alcanivorax & 0.3755 & 0.0686 & 0.5066 & 0.0827 & 0.0917 & 0.2390 & 0.0360 \\
\hline P_Proteobacteria;c_Gammaproteobacteria;o_Oceanospirillales;f_Halomonadaceae;g_Halomonas & 0.5041 & 0.1557 & 0.3472 & 0.2894 & 0.2385 & 0.3983 & 0.8015 \\
\hline P_Proteobacteria;c_Gammaproteobacteria;o_Oceanospirillales;f_Oceanospirillaceae;g_Marinobacterium & 2.3481 & 3.7716 & 2.5613 & 7.9183 & $\begin{array}{r}19.537 \\
6\end{array}$ & 1.4826 & 3.1342 \\
\hline P_Proteobacteria;c_Gammaproteobacteria;o_Oceanospirillales;f_Oceanospirillaceae;g_Nitrincola & 0.0540 & 0.0290 & 0.0180 & 0.0284 & 0.0288 & 0.0103 & 0.0385 \\
\hline P_Proteobacteria;c_Gammaproteobacteria;o_Oceanospirillales;f_Oceanospirillaceae;g_Oleibacter & 1.6280 & 6.3952 & 1.0338 & 2.0597 & 1.7614 & 0.2313 & 0.3519 \\
\hline P_Proteobacteria;c_Gammaproteobacteria;o_Pseudomonadales;f_Moraxellaceae;g_Acinetobacter & 0.0926 & 0.4144 & 0.2932 & 0.0517 & 0.0498 & 0.1490 & 0.3648 \\
\hline p_Proteobacteria;c_Gammaproteobacteria;o_Pseudomonadales;f_Moraxellaceae;g_Enhydrobacter & 0.0000 & 0.0000 & 0.0000 & 0.0000 & 0.0000 & 0.0051 & 0.0077 \\
\hline p_Proteobacteria;c_Gammaproteobacteria;o_Pseudomonadales;f_Moraxellaceae;g_Psychrobacter & 0.0000 & 0.0000 & 0.0000 & 0.0026 & 0.0000 & 0.0000 & 0.0077 \\
\hline P_Proteobacteria;c_Gammaproteobacteria;o_Pseudomonadales;f_Pseudomonadaceae;g_Azorhizophilus & 0.0026 & 0.0185 & 0.0103 & 0.0129 & 0.0157 & 0.0026 & 0.0154 \\
\hline P_Proteobacteria;c_Gammaproteobacteria;o_Pseudomonadales;f_Pseudomonadaceae;g_Pseudomonas & 1.7514 & 1.1983 & 1.3604 & 1.5454 & 3.0510 & 1.1537 & 5.2715 \\
\hline P_Proteobacteria;c_Gammaproteobacteria;o_Thiotrichales;__Piscirickettsiaceae;g_Methylophaga & 0.0026 & 0.0000 & 0.0077 & 0.0078 & 0.0052 & 0.0591 & 0.1927 \\
\hline P_Proteobacteria;c_Gammaproteobacteria;o_Vibrionales;f_Vibrionaceae;g_Vibrio & 0.0051 & 0.0026 & 0.0283 & 0.0155 & 0.0079 & 0.0000 & 0.0128 \\
\hline P_Proteobacteria;c_Gammaproteobacteria;o_Xanthomonadales;f_Sinobacteraceae;g_Hydrocarboniphaga & 0.0026 & 0.0000 & 0.0051 & 0.0026 & 0.0052 & 0.0000 & 0.0000 \\
\hline p_Proteobacteria;c_Gammaproteobacteria;o_Xanthomonadales;f_Xanthomonadaceae;g_Arenimonas & 0.0000 & 0.0000 & 0.0000 & 0.0000 & 0.0000 & 0.1259 & 0.0077 \\
\hline P_Proteobacteria;c_Gammaproteobacteria;o_Xanthomonadales;f_Xanthomonadaceae;g_Luteimonas & 0.0077 & 0.0000 & 0.0000 & 0.0000 & 0.0026 & 0.0000 & 0.0000 \\
\hline P_Proteobacteria;c_Gammaproteobacteria;o_Xanthomonadales;f_Xanthomonadaceae;g_Lysobacter & 0.0000 & 0.0026 & 0.0026 & 0.0129 & 0.0000 & 0.0000 & 0.0000 \\
\hline $\begin{array}{l}\text { P_Proteobacteria;c_Gammaproteobacteria;o_Xanthomonadales;f_Xanthomonadaceae;g_Pseudoxanthomo } \\
\text { nas }\end{array}$ & 0.0000 & 0.0000 & 0.0000 & 0.0000 & 0.0000 & 0.3726 & 0.0283 \\
\hline P_Proteobacteria;c_Gammaproteobacteria;o_Xanthomonadales;__Xanthomonadaceae;g_Rhodanobacter & 0.0077 & 0.0000 & 0.0000 & 0.0052 & 0.0026 & 0.0128 & 0.0308 \\
\hline $\begin{array}{l}\text { p_Proteobacteria;c_Gammaproteobacteria;o_Xanthomonadales;f_Xanthomonadaceae;g_Stenotrophomona } \\
\text { s }\end{array}$ & 0.0077 & 0.0396 & 0.0206 & 0.0594 & 0.0210 & 0.0051 & 0.0103 \\
\hline P_Spirochaetes;c_[Leptospirae];o_[Leptospirales];f_Sediment-4;g_SJA-88 & 0.0000 & 0.0132 & 0.0000 & 0.0026 & 0.0000 & 0.0000 & 0.0000 \\
\hline P_Spirochaetes;c_Spirochaetes;o_Sphaerochaetales;f_Sphaerochaetaceae;g_Sphaerochaeta & 4.6884 & 4.5450 & 3.5591 & 0.3463 & 4.1177 & 0.0385 & 0.4290 \\
\hline P_Spirochaetes;c_Spirochaetes;o_Spirochaetales;f_Spirochaetaceae;g_Treponema & 0.0411 & 0.1452 & 0.0977 & 0.0594 & 0.3853 & 0.1876 & 0.0231 \\
\hline P_Spirochaetes;c_WWE1;o_[Cloacamonales];f_[Cloacamonaceae];g_W22 & 0.2032 & 8.9712 & 0.1569 & 0.3902 & 0.6553 & 0.0154 & 0.0051 \\
\hline P_Synergistetes;c_Synergistia;o_Synergistales;f_Aminiphilaceae;g_Aminiphilus & 0.0026 & 0.0000 & 0.0051 & 0.0052 & 0.0000 & 0.0103 & 0.0257 \\
\hline P_Synergistetes;c_Synergistia;o_Synergistales;f_Anaerobaculaceae;g_Anaerobaculum & 0.0514 & 0.0026 & 0.0463 & 0.0026 & 0.0026 & 0.0000 & 0.0000 \\
\hline P_Synergistetes;c_Synergistia;o_Synergistales;f_Dethiosulfovibrionaceae;g_Aminobacterium & 0.1003 & 0.0079 & 0.0386 & 0.0543 & 0.0157 & 0.0000 & 0.0334 \\
\hline P_Synergistetes;c_Synergistia;o_Synergistales;f_Dethiosulfovibrionaceae;g_Dethiosulfovibrio & 0.0129 & 0.0475 & 0.0026 & 0.0284 & 0.0026 & 0.0488 & 0.0154 \\
\hline P_Synergistetes;c_Synergistia;o_Synergistales;f_Dethiosulfovibrionaceae;g_HA73 & 1.4222 & 0.8103 & 0.6198 & 0.7546 & 0.4744 & 0.1079 & 0.1850 \\
\hline P_Synergistetes;c_Synergistia;o_Synergistales;f_Synergistaceae;g_vadinCA02 & 0.2186 & 3.7426 & 0.2520 & 3.9824 & 0.4089 & 0.0463 & 0.2029 \\
\hline P_Synergistetes;c_Synergistia;o_Synergistales;f_Thermovirgaceae;g_Thermovirga & 0.0051 & 0.0026 & 0.0000 & 0.0052 & 0.0026 & 0.0000 & 0.0000 \\
\hline P_Tenericutes;c_Mollicutes;o_Acholeplasmatales;_f_Acholeplasmataceae;g_Acholeplasma & 0.3215 & 2.4995 & 0.3446 & 0.2998 & 1.4704 & 0.0180 & 0.0128 \\
\hline P_Tenericutes;c_Mollicutes;o_Anaeroplasmatales;f_Anaeroplasmataceae;g_RFN20 & 0.0103 & 0.0739 & 0.0103 & 0.0103 & 0.0262 & 0.0000 & 0.0000 \\
\hline P_Thermi;c_Deinococci;o_Thermales;f_Thermaceae;g_Thermus & 0.0026 & 0.0053 & 0.0026 & 0.0000 & 0.0052 & 0.0026 & 0.0026 \\
\hline P_Thermotogae;c_Thermotogae;o_Thermotogales;f_Thermotogaceae;g_Kosmotoga & 0.0463 & 0.3273 & 0.0360 & 0.0413 & 0.5006 & 0.1567 & 0.0771 \\
\hline P_Thermotogae;c_Thermotogae;o_Thermotogales;f_Thermotogaceae;g_Petrotoga & 0.0077 & 0.0026 & 0.0000 & 0.0026 & 0.0000 & 0.0000 & 0.0000 \\
\hline p_Verrucomicrobia;c_Opitutae;o_Opitutales;f_Opitutaceae;g_Opitutus & 0.1234 & 0.0079 & 0.2057 & 0.0233 & 0.0157 & 0.0231 & 0.5241 \\
\hline & 27.778 & 45.542 & 25.034 & 30.541 & 55.761 & 54.309 & 56.473 \\
\hline Total percent & 2 & 1 & 7 & 4 & 2 & 0 & 8 \\
\hline
\end{tabular}

